\title{
Relationships Between Renin, Aldosterone, and 24-Hour Ambulatory Blood Pressure in Obese Adolescents
}

\author{
IBRAHIM F. SHATAT AND JOSEPH T. FLYNN \\ Division of Pediatric Nephrology [I.F.S.], The Medical University of South Carolina Children's Hospital, Charleston, \\ South Carolina 29425; Division of Nephrology [J.T.F.], Seattle Children's Hospital, Seattle, Washington 98105
}

\begin{abstract}
Renin-angiotensin system (RAS) activation and abnormalities of ambulatory blood pressure $(\mathrm{ABP})$ are present in obesity, but relationships between components of the RAS and ABP have not been defined in the young. Anthropometric measurements and 24-h ABP were obtained on 30 obese adolescents with and without type 2 diabetes mellitus. Plasma renin activity (PRA), aldosterone, and other cardiovascular risk factors were measured. Median PRA levels were 2.5 [interquartile range (IQR), 1.7-4.1] ng/mL/h and were higher in the diabetic subjects compared with the nondiabetics. Females had significantly higher PRA than males 3.2 (IQR, $2-4.8)$ versus 1.8 (IQR, 1.1-2.9) $\mathrm{ng} / \mathrm{mL} / \mathrm{h}(p=0.04)$ and were more obese. BMI $Z$ score and PRA were significantly correlated (rho = $0.46, p<0.001)$. PRA inversely correlated with 24-h systolic ABP (rho $=-0.46, p=0.02$ ) and strongly with 24-h pulse pressure (rho $=-0.61, p=0.001$ ). Aldosterone levels were also correlated with 24-h pulse pressure (rho $=-0.46, p=0.02$ ). In multivariate models, lower PRA was independently associated with 24-h systolic blood pressure. In this study, PRA was positively correlated with BMI, but the relationships between components of the RAS and ABP were inverse. Further studies are needed to define the pathophysiologic relationship between RAS components and blood pressure regulation in obese youth. (Pediatr Res 69: 336-340, 2011)
\end{abstract}

$\mathrm{T}$ he renin-angiotensin system (RAS) plays a central role in blood pressure (BP) regulation and fluid-electrolyte homeostasis, and its dysregulation contributes to the development of hypertension (1). Activation of the RAS in adipose tissue may represent an important link between obesity and hypertension. Studies have demonstrated that rat and human adipose tissue possess all of the components necessary for production of angiotensin II (Ang II), including angiotensinogen (2), renin-like activity (3), Ang-converting enzyme, and Ang type 1 receptor $(4,5)$. Adipose-derived angiotensinogen is also released into the circulation where it serves as substrate for conversion to bioactive Ang II, contributes to plasma Ang II levels, and increases BP (6).

Other metabolically active products of adipose tissue include adipokines. We have previously shown that adiponectin (an anti-inflammatory, anti-atherosclerotic adipokine) levels are inversely related to ambulatory blood pressure (ABP) (7), which is itself abnormal in obesity.

Received June 11, 2010; accepted November 14, 2010.

Correspondence: Ibrahim F. Shatat, M.D., Assistant Professor of Pediatrics, Division of Nephrology, CSB-316, MUSC Children's Hospital, 96 Jonathan Lucas Street, Charleston, SC 29425; e-mail: shatat@musc.edu

Supported by Grant 4272387 from the Kidney and Urology Foundation of America [I.F.S.].
Whether components of the RAS have similar relationships to ABP in obese youth is unknown. Our objective in this study was to examine the relationships between 24-h BP measurements and plasma renin activity (PRA) and aldosterone levels in obese adolescents.

\section{MATERIALS AND METHODS}

Setting and study design. Patients who fulfilled the following criteria were recruited from the Pediatric Diabetes and Pediatric Nephrology Clinics at the Children's Hospital at Montefiore in the Bronx, NY: age 10 to $21 \mathrm{y}$, obesity defined as BMI $>95$ th percentile for age and gender (8), a diagnosis within the past 3 y of type 2 diabetes mellitus (T2DM) according to the standard American Diabetes Association criteria (9), and serum test results negative for antibodies to glutamic acid decarboxylase-65 or insulin autoantibodies. Obese nondiabetic subjects, defined as BMI $>95$ th percentile for age and gender, and normal glucose tolerance, were recruited from primary care, nephrology, and pediatric endocrinology clinics.

T2DM patients were ineligible when they were metabolically unstable, as defined by the history of an episode of diabetic ketoacidosis or of hospitalization for uncontrolled diabetes within the previous 2 mo. Patients with comorbid inflammatory disease, secondary hypertension, other endocrine disorders, or any genetic syndrome that would independently predispose them to either diabetes mellitus or to kidney disease were also excluded. In addition, patients on treatment with insulin sensitizing agents with potential effect on BP (10), statins and any antihypertensive medication including angiotensin converting enzyme inhibitors and angiotensin receptor blockers (ARB) were excluded.

Written informed consent was obtained from the parent or guardian of each subject. Subjects who were 13 - to 21 -y-old also provided written informed consent. Written informed assent was obtained from children aged 10 to $13 \mathrm{y}$. The protocol was approved by the Montefiore Medical Center Institutional Review Board.

Study procedures and definitions. Subjects had a study visit that included a history and a physical examination. Family health history, smoking status, and medication and vitamin usage were assessed. Families self-identified the race/ethnicity of the subject.

Anthropometric and body composition measurements were performed in the morning, before breakfast, with the subject wearing light clothing, without footwear. Body weight and standing height were measured by the same observer using a digital scale and a wall-mounted stadiometer to the nearest $0.1 \mathrm{~kg}$ and $0.5 \mathrm{~cm}$, respectively. BMI was calculated by dividing the weight in kilograms by the square of the height in meters and further used to calculate the BMI $Z$ score.

Three resting BP measurements were obtained from the right upper arm using an aneroid sphygmomanometer and appropriate size cuff (11). The first measurement was discarded and the average of the other two measurements was recorded as the study visit BP. Subjects were classified as having casual

ABBREVIATIONS: ABP, ambulatory blood pressure; Ang, angiotensin; BP, blood pressure; DBP, diastolic blood pressure; HOMA-IR, homeostasis model assessment of insulin resistance; hsCRP, high sensitivity $\mathrm{C}$ reactive protein; IQR, interquartile range; PRA, plasma renin activity; RAS, reninangiotensin system; SBP, systolic blood pressure; T2DM, type 2 diabetes mellitus 
hypertension if this mean value was $\geq$ the 95 th percentile for age, gender, and height (11).

The subjects underwent a 24-h ABP recording using the Spacelabs 90217 ambulatory monitor (Spacelabs Medical, Issaquah, WA), fitted, and programmed as previously described $(7,12)$. Awake, or daytime, and sleep, or nocturnal, periods were determined by means of a diary provided by the subjects. Ambulatory hypertension was diagnosed when the average ambulatory systolic blood pressure (SBP) or diastolic blood pressure (DBP) for the period was $\geq 95$ th percentile BP on the basis of the subject's sex and height according to normative values for ABP (13). BP load was defined as the percentage of readings for a given period that exceeded the 95th percentile for that individual. Percent dipping was calculated for both average SBP and DBP with the following formula: [(daytime BP - nocturnal BP $/$ daytime $\mathrm{BP}] \times$ 100. Each subject was categorized as a "dipper" (decrease in average SBP and $\mathrm{DBP} \geq 10 \%$ during sleep) or a "nondipper" (decrease $<10 \%$ ) (14). ABP index (mean ABP divided by the 95th percentile for pediatric ABP) was also calculated.

A fasting blood sample was drawn for PRA, aldosterone level, glucose, insulin, high sensitivity $\mathrm{C}$ reactive protein (hsCRP), creatinine, and cholesterol levels. Urinary creatinine and microalbumin measurements were obtained from two random mid-stream urinary specimens obtained from all subjects. All routine clinical tests, except for PRA and aldosterone levels, were performed in the Montefiore Medical Center's clinical laboratory.

Microalbuminuria was defined as an albumin-to-creatinine ratio ranging between 30 and $300 \mathrm{mg} / \mathrm{g}$ creatinine (15). Homeostasis model assessment of insulin resistance (HOMA-IR) was calculated using the formula [fasting serum insulin $(\mu \mathrm{U} / \mathrm{mL}) \times$ fasting serum glucose $(\mathrm{mg} / \mathrm{dL})] \times 0.002246(16)$. The estimated GFR (eGFR) was calculated with the Schwartz formula (17)

PRA (ng/mL/h) and aldosterone levels (ng/dL) were measured at Quest Diagnostics using angiotensin I generation, RIA, analytical sensitivity: 0.37 $\mathrm{ng} / \mathrm{mL} / \mathrm{h}$ and liquid chromatography tandem mass spectrometry (LC/MS/MS),

Table 1. Demographic characteristics of study population

\begin{tabular}{|c|c|c|c|c|}
\hline Variable & $\begin{array}{l}\text { All patients } \\
\quad(n=30)\end{array}$ & $\begin{array}{c}\text { Obese } \\
(n=17)\end{array}$ & $\begin{array}{c}\text { Obese }+ \\
\text { T2DM } \\
(n=13)\end{array}$ & $p^{*}$ \\
\hline Age (y) & $14.4 \pm 2.4$ & $14.0 \pm 2.4$ & $14.9 \pm 2.3$ & NS \\
\hline Sex & & & & NS \\
\hline Male & $13(43 \%)$ & 9 & 4 & \\
\hline Female & $17(57 \%)$ & 8 & 9 & \\
\hline Race/ethnicity & & & & NS \\
\hline African American & $15(50 \%)$ & 8 & 7 & \\
\hline Hispanic & $15(50 \%)$ & 9 & 6 & \\
\hline BMI, $\mathrm{kg} / \mathrm{m}^{2}$ & $35.4 \pm 6.0$ & $34.7 \pm 5.9$ & $36.1 \pm 6.2$ & NS \\
\hline Clinic BP $\ddagger$ & & & & NS \\
\hline Normotensive & $10(30 \%)$ & 6 & 4 & \\
\hline Prehypertensive & $5(20 \%)$ & 3 & 2 & \\
\hline Hypertensive & $15(50 \%)$ & 8 & 7 & \\
\hline Ambulatory $\mathrm{BP} \ddagger$ & & & & NS \\
\hline Normotensive & $26(87 \%)$ & 14 & 12 & \\
\hline Hypertensive & $4(13 \%)$ & 3 & 1 & \\
\hline
\end{tabular}

Categorical variables presented as $n(\%)$, with $p$ values calculated by Fisher's exact test. Normally distributed continuous variables are presented as mean $\pm \mathrm{SD}$, with $p$ values calculated by Student's $t$ test.

* Obese vs. the obese \pm T2DM groups.

$\dagger$ Classification based on Ref. 11.

\$ Classification based on Ref. 13 . analytical sensitivity: $1.0 \mathrm{ng} / \mathrm{dL}$, respectively. All subjects had their blood samples drawn in the seated position.

Data analysis. Categorical variables are presented as relative frequencies, and their association with obese subgroups were tested for significance using Fisher's Exact tests. Continuous variables, not normally distributed, are presented as medians and interquartile ranges (IQRs), and associations with subgroups were tested for significance using the Mann-Whitney $U$ test. Normally distributed continuous variables are presented as means \pm standard deviations, with differences between subgroups were assessed by two-tailed $t$ tests. Bivariate correlations between various measures of BP indices and PRA levels were examined using Spearman correlation.

Variables with significant correlations with the outcome variable (BP parameter) of magnitude $\geq 0.2$ were selected for the initial multivariate linear regression model, along with those variables considered clinically important or potential effect modifiers. Given that the two obese groups were comparable in many demographic, biochemical, and BP parameters, the two groups were treated as one group when building the regression model.

Statistical and database software used included STATA 9.2 (Stata Corporation, College Station, TX) and Microsoft Office Excel 2003 (Microsoft Corporation, Redmond, WA), respectively. Statistical significance for all analyses was set at two-sided $p<0.05$.

\section{RESULTS}

Study population. Thirty obese adolescents were studied; 17 were nondiabetic (mean age, $14.0 \pm 2.4 \mathrm{y}$ ) and $13 \mathrm{had}$ T2DM (14.9 \pm 2.3 y). Mean BMI in both groups was $35.4 \pm$ $6.0 \mathrm{~kg} / \mathrm{m}^{2}$. Notably, both the females and the obese group with T2DM had higher BMI compared with males and obese nondiabetic subjects, respectively. Fifty-seven percent were female, 50\% percent were African American (AA), and 50\% were Hispanic. Fifty percent were classified as hypertensive based on their clinic BP measurements (Table 1).

Biochemical parameters. Laboratory results are displayed in Table 2. Compared with published normative data (18), median PRA was low normal in our obese cohort, 2.5 (IQR, 1.7-4.1) $\mathrm{ng} / \mathrm{mL} / \mathrm{h}$. PRA levels were higher but not significantly different in the diabetic subjects 2.7 (IQR, 2.4-4.2) $\mathrm{ng} / \mathrm{mL} / \mathrm{h}$ compared with the nondiabetics 2.0 (IQR, 1.6-3.4) $\mathrm{ng} / \mathrm{mL} / \mathrm{h}$. Females had significantly higher PRA than males $3.2(2-4.8)$ versus $1.8(1.1-2.9) \mathrm{ng} / \mathrm{mL} / \mathrm{h}(p=0.04)$. No racial differences in PRA were observed.

The median aldosterone level in this population $(6 \mathrm{ng} / \mathrm{dL}$; IQR, 2-11.5 ng/dL) fell within the range of published normative data (19); however, levels were significantly higher in diabetic subjects compared with nondiabetics (Table 2, $p=$ 0.05 ). Obese diabetics also tended to have higher hsCRP levels and statistically different HOMA-IR and urinary microalbumin compared with obese nondiabetics.

24-h ABP measurements and relationship to biochemical parameters. Table 3 displays the 24-h ABP measurements in our study population. Four subjects (13\%) were classified to have ambulatory hypertension. Mean SBP and DBP dip were

Table 2. Biochemical parameters

\begin{tabular}{|c|c|c|c|c|}
\hline Variable & All patients $(n=30)$ & Obese $(n=17)$ & Obese + T2DM $(n=13)$ & $p$ \\
\hline Plasma renin activity, $\mathrm{ng} / \mathrm{mL} / \mathrm{h}^{*}$ & $2.5(1.7-4.1)$ & $2.0(1.6-3.4)$ & $2.7(2.4-4.2)$ & NS \\
\hline Aldosterone, ng/dL $\ddagger$ & $6(2-11.5)$ & $4(2,7)$ & $10(5-14)$ & 0.05 \\
\hline hsCRP, mg/dL & $0.26(0.12-0.6)$ & $0.21(0.11-0.5)$ & $0.4(0.2-0.72)$ & NS \\
\hline HOMA-IR & $6.0(3.6-11.4)$ & $4.4(3.0-5.5)$ & $11.1(8.0-30.0)$ & $<0.01$ \\
\hline Total cholesterol, mg/dL & $159.8 \pm 32.5$ & $157.9 \pm 33.3$ & $162.2 \pm 32.5$ & NS \\
\hline Microalbumin:creatinine ratio, $\mathrm{mg} / \mathrm{g}$ & $18.2(10.7-69.2)$ & $15.7(9.4-21.9)$ & $69.2(12.1-174.8)$ & 0.03 \\
\hline
\end{tabular}

\footnotetext{
* Reference laboratory normal range $\leq 16.0 \mathrm{ng} / \mathrm{mL} / \mathrm{h}(24)$.
}

$\dagger$ Reference laboratory normal range $\leq 35 \mathrm{ng} / \mathrm{dL}$ (25). 
Table 3. 24-h ABP measurements

\begin{tabular}{|c|c|c|c|c|}
\hline $\mathrm{ABPM}$ variable & All patients & Obese & Obese + T2DM & $p$ \\
\hline SBP index* & $0.9 \pm 0.09$ & $0.9 \pm 0.09$ & $0.9 \pm 0.08$ & NS \\
\hline DBP index* & $0.83 \pm 0.07$ & $0.81 \pm 0.06$ & $0.86 \pm 0.08$ & 0.07 \\
\hline SBP dip, $\%$ & $8.5 \pm 5.7$ & $8.7 \pm 4.8$ & $8.2 \pm 7.1$ & NS \\
\hline DBP dip, $\%$ & $13.6 \pm 8.3$ & $12.0 \pm 7.8$ & $16.0 \pm 8.8$ & NS \\
\hline SBP load, \% & $18.6(2.8-46.9)$ & $23.8(3.8-53.7)$ & $15.78(1.0-40.2)$ & NS \\
\hline DBP load, \% & $4(2.8-10.8)$ & $4(2.4-4.9)$ & $4.9(3.0-12.3)$ & 0.1 \\
\hline
\end{tabular}

* Subject's result divided by the 95th percentile value for child of same age, gender, and height. ABPM, ambulatory blood pressure monitoring.

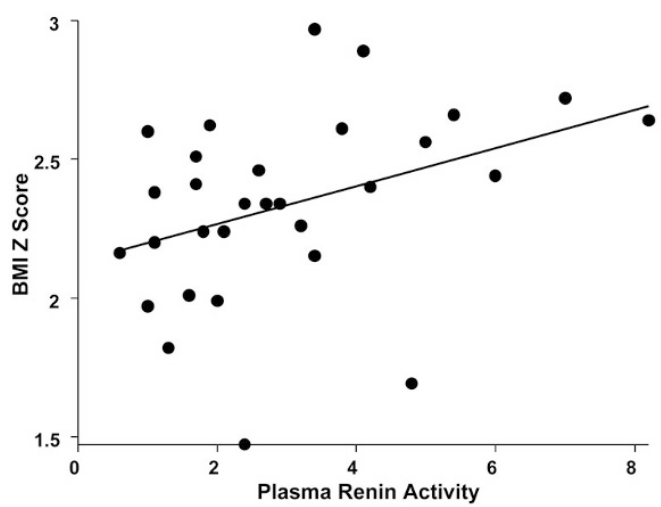

Figure 1. Significant positive correlation between BMI $Z$ score and PRA, rho $=0.46, p<0.01$.
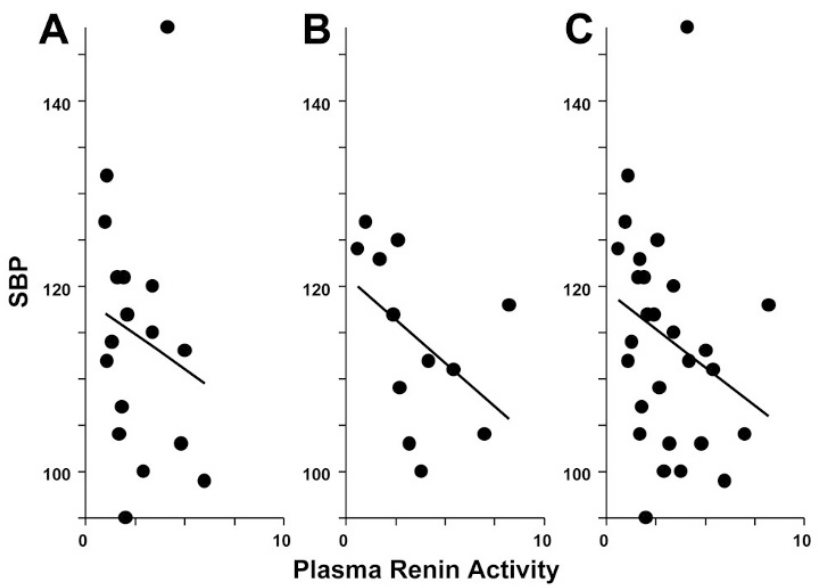

Figure 2. Inverse correlation between SBP and PRA. (A) obese nondiabetic; $(B)$ obese diabetic; $(C)$ all study participants.

$8.5 \pm 5.7 \%$ and $13.6 \pm 8.3 \%$, respectively, with 17 study participants (57\%) who had impaired SBP dipping and 9 (30\%) who had impaired DBP dipping.

PRA inversely and significantly correlated with 24-h SBP (rho $=-0.44, p=0.02)$, but not with the 24-h DBP $($ rho $=$ $0.10, p=0.59$ ). Figures 1 and 2 demonstrate the significant positive correlations between BMI $Z$ score and PRA, and the inverse correlation between SBP and PRA, respectively. Aldosterone levels were not significantly associated with ABP parameters. Pulse pressure negatively correlated with PRA and aldosterone levels, $r=-0.61, p<0.001$ and $r=-0.46$, $p=0.02$, respectively (Fig. 3).

Determinants of 24-h SBP. In a multivariable linear regression model (Table 4), lower PRA levels and higher BMI
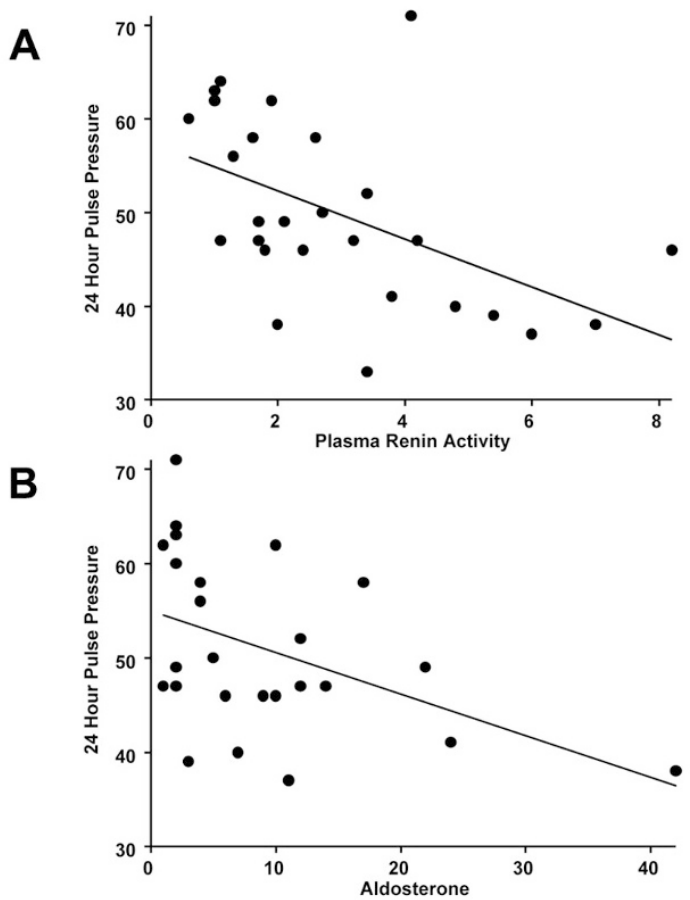

Figure 3. Inverse correlation between 24-h pulse pressure and (A) PRA levels $(r=-0.61, p<0.001)$ and $(B)$ aldosterone levels $(r=-0.46, p=0.02)$.

Table 4. Multivariable linear regression for determinants of 24-h SBP

\begin{tabular}{lcl}
\hline \multicolumn{1}{c}{ Parameter } & Regression coefficients $(95 \%$ CI) & $p$ \\
\hline PRA, ng/mL/h & $-0.025(-0.043$ to -0.006$)$ & 0.012 \\
BMI Z score & $0.125(-0.001$ to 0.251$)$ & 0.05 \\
HOMA-IR & $0.001(-0.001$ to 0.003$)$ & 0.33 \\
hsCRP, mg/dL & $0.043(-0.002$ to 0.087$)$ & 0.06 \\
\hline
\end{tabular}

$\mathrm{Z}$ scores were independent determinants of higher 24-h SBP, with a reduction of $0.025 \mathrm{ng} / \mathrm{mL} / \mathrm{h}$ in PRA associated with a $1 \mathrm{~mm} \mathrm{Hg}$ increase in SBP. HOMA-IR was included in the final model for its clinical relevance and possible confounding effects, as well as hsCRP, which is a known independent determinant of cardiovascular risk (20).

\section{DISCUSSION}

It is now apparent from a growing body of literature that adipose tissue functions as an endocrine organ capable of interacting with and modifying important physiologic functions. In this study, we demonstrate that in obese adolescents, lower PRA is an independent determinant of 24-h SBP. We 
also demonstrate for the first time in a young cohort of obese adolescents that low PRA levels are significantly correlated with wide pulse pressure, a known predictor of cardiovascular mortality in adults (21), which has also been shown to independently associate with increased carotid intima-media thickness in adults exposed to large pulse pressure in adolescence (22). In addition, we describe a positive and significant correlation between PRA and severity of obesity, with more severely obese adolescents having higher PRA.

Abnormal BP in the state of obesity has been attributed to several intertwining factors, including activation of the sympathetic nervous system, hyperinsulinemia, chronic inflammation and cytokine overproduction, obstructive sleep apnea, hyperlipidemia and accelerated atherosclerosis, and more recently activation of the RAS $(23,24)$. The contribution of adipose tissue renin to circulating renin levels is not well understood. PRA levels in our cohort were in the low-normal range, suggesting that adipose tissue contribution to $\mathrm{BP}$ regulation is limited and possibly modified by other obesity related mechanisms.

Some of these factors may contribute to the altered sodium handling and fluid and salt retention seen in obesity (25) and may possibly explain low normal PRA levels as a result of volume expansion in this population. Another possible explanation of low PRA levels is the marked increase in plasma Ang II concentrations in diet induced obese rats reported by Boustany et al. (26), with this marked increase in circulating Ang II concentration, kidney renin mRNA expression and PRA would be anticipated to decrease from negative feedback (27).

Aldosterone and/or activation of the mineralocorticoid receptor likely play a significant role in the pathogenesis of obesity hypertension. This is supported by data demonstrating a significant BP reduction with spironolactone treatment in obese individuals thought to be resistant to other antihypertensives (28). In addition, positive relationships between aldosterone-to-renin ratio (ARR), aortic BP, and arterial stiffness have been demonstrated in other patient populations (29). Consistent with previous studies in the pediatric age group (30), we did not find a significant correlation between this ratio and 24-h SBP or pulse pressure in this study. Furthermore, ARR did not remain as an independent determinant in the multivariate regression model with $24-\mathrm{h}$ SBP as the outcome variable.

On the other hand, our finding that aldosterone levels were significantly higher in the obese diabetics is intriguing. An association has been demonstrated in animal models between elevated aldosterone, type 2 diabetes, and the development of diabetic nephropathy (31). This finding in combination with our previous work showing a significant prevalence of microalbuminuria in adolescents with type 2 diabetes (12) suggests that aldosterone receptor blockade may have an important role in the treatment of hypertension in this patient population. Additional studies are warranted to further explore this relationship.

Our study was limited by its small sample size; this may have affected which variables could be included in the final model based on their significance level. The cross-sectional study design does not allow a cause-and-effect relationship to be established. In addition, the racial composition of the study population was limited to obese minorities, which may limit the generalizability of our findings. In addition, it is possible that other factors, not accounted for in this study, such as salt intake and obstructive sleep apnea might have contributed to the BP levels and the high rate of impaired nocturnal dipping observed in this study. However, the study also has unique strengths, especially the use of ambulatory BP monitoring, which provides superior assessment of $\mathrm{BP}$ compared with office measurements (18).

Obesity in childhood is a growing heath care burden and is often complicated by the development of hypertension. BP regulation in obese subjects is complex and not fully understood. In this study, PRA levels were in the low-normal range and correlated with BMI and inversely with 24-h SBP and pulse pressure. Further studies are needed to define the pathophysiologic relationship between different RAS components and $\mathrm{BP}$ regulation in the state of obesity.

\section{REFERENCES}

1. Kim S, Iwao H 2000 Molecular and cellular mechanisms of angiotensin II-mediated cardiovascular and renal diseases. Pharmacol Rev 52:11-34

2. Engeli S, Gorzelniak K, Kreutz R, Runkel N, Distler A, Sharma AM 1999 Coexpression of renin-angiotensin system genes in human adipose tissue. J Hypertens 17:555-560

3. Shenoy U, Cassis L 1997 Characterization of renin activity in brown adipose tissue. Am J Physiol 272:C989-C999

4. Schling P, Mallow H, Trindl A, Loffler G 1999 Evidence for a local renin angiotensin system in primary cultured human preadipocytes. Int J Obes Relat Metab Disord 23:336-341

5. Ye ZW, Wu XM, Jiang JG 2009 Expression changes of angiotensin II pathways and bioactive mediators during human preadipocytes-visceral differentiation. Metabolism 58:1288-1296

6. Massiéra F, Bloch-Faure M, Ceiler D, Murakami K, Fukamizu A, Gasc JM, Quignard-Boulange A, Negrel R, Ailhaud G, Seydoux J, Meneton P, Teboul M 2001 Adipose angiotensinogen is involved in adipose tissue growth and blood pressure regulation. FASEB J 15:2727-2729

7. Shatat IF, Freeman KD, Vuguin PM, Dimartino-Nardi JR, Flynn JT 2009 Relationship between adiponectin and ambulatory blood pressure in obese adolescents. Pediatr Res 65:691-695

8. Reilly JJ, Dorosty AR, Emmett PM; Avon Longitudinal Study of Pregnancy and Childhood Study Team 2000 Identification of the obese child: adequacy of the body mass index for clinical practice and epidemiology. Int J Obes Relat Metab Disord 24:1623-1627

9. American Diabetes Association 2003 Standards of medical care for patients with diabetes mellitus. Diabetes Care 26:S33-S50

10. Kotchen TA 1996 Attenuation of hypertension by insulin-sensitizing agents. Hypertension 28:219-223

11. National High Blood Pressure Education Program Working Group on High Blood Pressure in Children and Adolescents 2004 The fourth report on the diagnosis, evaluation, and treatment of high blood pressure in children and adolescents. Pediatrics 114:555-576

12. Ettinger LM, Freeman K, DiMartino-Nardi JR, Flynn JT 2005 Microalbuminuria and abnormal ambulatory blood pressure in adolescents with type 2 diabetes mellitus. J Pediatr 147:67-73

13. Urbina E, Alpert B, Flynn J, Hayman L, Harshfield GA, Jacobson M, Mahoney L, McCrindle B, Mietus-Snyder M, Steinberger J, Daniels S 2008 Ambulatory blood pressure monitoring in children and adolescents: recommendations for standard assessment: a scientific statement from the American Heart Association Atherosclerosis, Hypertension, and Obesity in Youth Committee of the council on cardiovascular disease in the young and the council for high blood pressure research. Hypertension 52:433-451

14. Verdecchia P, Schillaci G, Guerrieri M, Gatteschi C, Benemio G, Boldrini F, Porcellati C 1990 Circadian blood pressure changes and left ventricular hypertrophy in essential hypertension. Circulation 81:528-536

15. Babazono T, Takahashi C, Iwamoto Y 2004 Definition of microalbuminuria in first-morning and random spot urine in diabetic patients. Diabetes Care 27:18381839

16. Matthews DR, Hosker JP, Rudenski AS, Naylor BA, Treacher DF, Turner RC 1985 Homeostasis model assessment: insulin resistance and beta-cell function from fasting plasma glucose and insulin concentrations in man. Diabetologia 28:412-419

17. Schwartz GJ, Haycock GB, Edelmann CM Jr, Spitzer A 1976 A simple estimate of glomerular filtration rate in children derived from body length and plasma creatinine. Pediatrics 58:259-263 
18. Stalker HP, Holland NH, Kotchen JM, Kotchen TA 1976 Plasma renin activity in healthy children. J Pediatr 89:256-258

19. Lashansky G, Saenger P, Dimartino-Nardi J, Gautier T, Mayes D, Berg G, Reiter E 1992 Normative data for the steroidogenic response of mineralocorticoids and their precursors to adrenocorticotropin in a healthy pediatric population. J Clin Endocrinol Metab 75:1491-1496

20. Blake GJ, Rifai N, Buring JE, Ridker PM 2003 Blood pressure, C-reactive protein, and risk of future cardiovascular events. Circulation 108:2993-2999

21. Fang J, Madhavan S, Alderman MH 2000 Pulse pressure: a predictor of cardiovascular mortality among young normotensive subjects. Blood Press 9:260-266

22. Raitakari OT, Juonala M, Taittonen L, Jula A, Laitinen T, Kähönen M, Viikari JS 2009 Pulse pressure in youth and carotid intima-media thickness in adulthood: the cardiovascular risk in young Finns study. Stroke 40:1519-1521

23. Hall JE 2003 The kidney, hypertension, and obesity. Hypertension 41:625-633

24. Sarzani R, Salvi F, Dessì-Fulgheri P, Rappelli A 2008 Renin-angiotensin system, natriuretic peptides, obesity, metabolic syndrome, and hypertension: an integrated view in humans. J Hypertens 26:831-843

25. Engeli S, Sharma AM 2001 The renin-angiotensin system and natriuretic peptides in obesity-associated hypertension. J Mol Med 79:21-29
26. Boustany CM, Bharadwaj K, Daugherty A, Brown DR, Randall DC, Cassis LA 2004 Activation of the systemic and adipose renin-angiotensin system in rats with diet-induced obesity and hypertension. Am J Physiol Regul Integr Comp Physiol 287:R943-R949

27. Schunkert H, Ingelfinger JR, Jacob H, Jackson B, Bouyounes B, Dzau VJ 1992 Reciprocal feedback regulation of kidney angiotensinogen and renin mRNA expressions by angiotensin II. Am J Physiol 263:E863-E869

28. Goodfriend TL, Calhoun DA 2004 Resistant hypertension, obesity, sleep apnea, and aldosterone: theory and therapy. Hypertension 43:518-524

29. Mahmud A, Feely J 2005 Aldosterone-to-renin ratio, arterial stiffness, and the response to aldosterone antagonism in essential hypertension. Am J Hypertens 18:50-55

30. Li R, Richey PA, DiSessa TG, Alpert BS, Jones DP 2009 Blood aldosterone-to-renin ratio, ambulatory blood pressure, and left ventricular mass in children. J Pediatr 155:170-175

31. Fredersdorf S, Endemann DH, Luchner A, Heitzmann D, Ulucan C, Birner C, Schmid P, Stoelcker B, Resch M, Muders F, Riegger GA, Weil J 2009 Increased aldosterone levels in a model of type 2 diabetes mellitus. Exp Clin Endocrinol Diabetes 117:15-20 